\title{
Retenedores de Ortodoncia y su Efecto sobre la Salud Periodontal. Revisión de la Literatura
}

\author{
Orthodontic Retainers and Their Effect On Periodontal Health. Literature Review
}

\author{
Cortínez Lira Cristine1; Figueroa Salinas Macarena1; Chamorro Pino Susana2; \\ Zafe Bastías Fadua1 \& Gallardo Sanzana Valentina²
}

CORTínEZ, L. C.; FIGUEROA, S. M.; CHAMORRO, P. S.; ZAFE, B. F. \& GALLARDO, S. V. Retenedores de ortodoncia y su efecto sobre la salud periodontal. Revisión de la literatura. Int. J. Odontostomat., 15(2):460-465, 2021.

RESUMEN: Existe una prescripción generalizada de retenedores fijos y removibles, con indicación indefinida, luego del tratamiento ortodóncico. El objetivo de este estudio fue dilucidar el impacto de los retenedores de ortodoncia sobre la salud periodontal de los dientes. Se realizó una búsqueda y análisis de artículos científicos de las bases de datos PubMed, ScienceDirect, SciELO, LILACS y Google Scholar. Los artículos seleccionados fueron publicados entre los años 2015 y 2020 en base a criterios de inclusión y exclusión. Nuestra revisión sugiere que la indicación de un retenedor fijo o removible presenta mayores beneficios que riesgos y no significa, cuando son consideradas las características individuales de cada paciente, el detrimento de la salud periodontal. Sin embargo, son necesarios nuevos estudios prospectivos, con un tiempo de seguimiento suficiente y la presencia de un grupo control, para poder extrapolar resultados y los potenciales daños asociados con los retenedores de ortodoncia.

PALABRAS CLAVE: retenedor ortodoncia, resorción ósea, periodoncia.

\section{INTRODUCCIÓN}

El éxito en un tratamiento de ortodoncia no debe centrarse sólo en conseguir una correcta oclusión y alineaciones dentarias, si no también, en la estabilidad a largo plazo de estos resultados. En la actualidad, el uso de retenedores posterior al tratamiento ortodóncico activo ha sido ampliamente difundido y se ha transformado en un procedimiento fundamental para mantener de las posiciones dentarias y prevenir la recidiva de maloclusiones.

Son numerosos los factores que pueden afectar la estabilidad de los resultados, por ejemplo, la tensión provocada por las fibras periodontales (fibras interdentales y dentogingivales), contactos oclusales no estables, cambios fisiológicos por la edad del paciente y la salud periodontal (Johnston et al., 2015). Esta etiología multifactorial lo vuelve un proceso difícil de predecir.

Existen variados tipos de retenedores que podemos clasificar en dos grupos principales: los removibles y fijos (Çifter et al., 2017). En ocasiones su uso es indicado de por vida, por lo que deben ser capaz de asegurar la salud periodontal de los dientes. No son muchos los estudios que relacionen ambos tópicos, por lo que la elección de uno u otro tipo de retenedor queda en muchas ocasiones supeditado a las preferencias personales de cada profesional (AlMoghrabi et al., 2016).

El objetivo de esta revisión de la literatura es dilucidar la relación que existe entre diferentes tipos de retenedores de ortodoncia utilizados en la actualidad y la salud periodontal en los pacientes.

\section{MATERIAL Y MÉTODO}

Se realizó una búsqueda electrónica de la literatura en las siguientes bases de datos: PubMed,

\footnotetext{
${ }^{1}$ Universidad Mayor, Santiago, Chile.

2 Universidad de Chile, Santiago, Chile.
} 
ScienceDirect, SciELO, LILACS, Google Scholar. Fueron utilizados los siguientes algoritmos en español e inglés:

- "Orthodontic Retainer" AND "Periodontal Health"

- "Orthodontic Retainer" AND "Periodontal Disease"

- "Retenedor de Ortodoncia" AND "Salud Periodontal"

- "Retenedor de Ortodoncia" AND "Enfermedad Periodontal"

Los criterios de inclusión fueron: artículos en idioma inglés o español, estudios en humanos, 5 años de antigüedad como máximo, disponibles en texto completo. Se consideraron como criterios de exclusión artículos en otros idiomas, basados en reportes de casos, estudios con pacientes con enfermedad periodontal previa, estudios realizados en animales y mayores a 5 años de antigüedad.

\section{RESULTADOS}

Se obtuvieron según la búsqueda realizada un total de 688 estudios científicos. Estos fueron tamizados por títulos y tras la eliminación de estudios duplicados, fuera de contexto, o que no cumplían criterios de inclusión o exclusión, se seleccionaron 18 para su evaluación a título y resumen, de los cuales 3 fueron excluidos. Finalmente, 15 estudios fueron seleccionados para el análisis a texto completo e incluidos para la revisión de la literatura.

\section{DISCUSIÓN}

El uso de retenedores o contenciones corresponde a la etapa final del tratamiento de ortodoncia cuyo objetivo es mantener los dientes en la posición correcta obtenida. Los dientes tienen tendencia a retornar a sus posiciones previas al tratamiento en un proceso que se ha descrito como multifactorial. Se describen como posibles causas las tensiones en las fibras periodontales que se insertan en el cuello del diente (fibras interdentales y dentogingivales), estabilidad de los contactos oclusales, cambios fisiológicos por la edad del paciente, cambios en los tejidos duros y blandos alrededor de las piezas dentarias (Johhnston et al.) (Di Venere et al., 2017). Se ha observado que las variaciones en las posiciones dentarias también ocurren en pacientes no tratado ortodóncicamente por lo que corresponde a un proceso altamente impredecible.
En una gran parte de los pacientes su uso es indicado de por vida por lo que se vuelve indispensable que sean compatibles con la salud periodontal. Los distintos retenedores pueden ser agrupados en dos tipos: removibles y fijos, en torno a ambos se han descrito diversas ventajas y desventajas (Storey et al., 2018).

Dentro de los retenedores removibles tenemos los Tipo Essix o estéticos que son de bajo costo, su fabricación en base a material termoplástico es rápida $y$, en general, tienen buena aceptación por el paciente. Sin embargo, no deben ser utilizados para comer ni beber alimentos por el riesgo de contacto continuo de las superficies dentales con alimentos cariogénicos. Otro tipo de retenedor removible son los Tipo Hawley, con la ventaja de poder ser utilizados para comer sin sufrir daños (Johnston et al.). En general, la desventaja de los retenedores removibles es que dependen de la disciplina y cooperación del paciente para su uso en el largo plazo (Storey et al.).

Los retenedores fijos, en general, son confeccionados con alambres de acero de distintos grosores, ya sea, trenzados doble o triple (adherido a todos los dientes anteriores) o de un solo alambre (adherido sólo a caninos). Si bien, muestran buenos resultados en cuanto a la prevención de recidiva han sido asociados a mayor acumulación de cálculo (Storey et al.), fallas adhesivas o fracturas del alambre (Gökçe \& Kaya, 2019). Por esta razón, existe preocupación sobre su impacto en el periodonto de las piezas dentarias. Durante su posicionamiento, el profesional debe ser prolijo y evitar el contacto de la resina compuesta con el margen gingival y espacio interdental (Johnston et al.; Di Venere et al.).

La enfermedad periodontal abarca tanto gingivitis como periodontitis, y a medida que progresa también lo hacen la destrucción de los tejidos de soporte del diente pudiendo llegar a provocar la pérdida de estos cuando es muy avanzada (Kinane, 2001; Highfield, 2009).

Los retenedores buscan mantener los dientes en la posición correcta obtenida posterior al tratamiento, pero al unirlos actúa como una férula, reduciendo los niveles de movilidad del diente lo que podría dañar el periodonto con el transcurso del tiempo (Westerlund et al., 2017).

En el año 2018 Storey et al. realizaron un estudio con dos grupos de pacientes correspondientes a 
30 con retenedores fijos a incisivos y caninos y, 30 con retenedores tipo Essix de arcada completa (superiores e inferiores para ambos grupos). Se evaluó la salud periodontal mediante el Índice de Placa (IP) e Índice Gingival (IG) de Silness and Loe, y el Índice de Cálculo (IC) de Greene and Vermillion. Como resultados se obtuvieron mayores niveles de IP, IC e IG con el uso de retención fija. Los dos últimos índices fueron estadísticamente significativos sólo en la zona intercanina. Sin embargo, no se pudo atribuir a los hallazgos alguna consecuencia importante sobre la salud periodontal como la pérdida de inserción clínica. Por otro lado, los resultados variaron según el centro en donde los pacientes fueron atendidos lo que puede ser relacionado con la motivación que realizó el especialista al momento de la instrucción de higiene oral.

Juloski et al. (2017) analizó la prevalencia de recesiones gingivales en dientes mandibulares cinco años posterior al tratamiento de ortodoncia. Comparó los resultados de tres grupos de pacientes: el primero tratados con retención fija mandibular, el segundo tratados sin retención indicada y un tercer grupo control. En todos los pacientes las recesiones gingivales fueron en aumento con el transcurso del tiempo y no se encontraron diferencias estadísticamente significativas intergrupos. Sin embargo, se encontró una mayor acumulación de cálculo dentario en la zona lingual mandibular en los pacientes con retención fija sin implicar detrimento de la salud periodontal. Por sí sola, la presencia de esta retención no parece predisponer a la resorción ósea. Dentro de las limitaciones del estudio encontramos que las mediciones se basaron en la inspección visual de modelos de yeso y fotografías. Los resultados concuerdan con los de Di Venere et al. quienes estudiaron pacientes a 36 meses de uso de retención fija, quienes no desarrollaron sacos periodontales o recesiones gingivales, por otro lado, aumentó el IC debido a mayor disponibilidad de sitios de retención ideales para la colonización bacteriana. Por esta razón, el especialista debe ser cuidadoso al momento de posicionar la contención, cuidando los excesos de resina compuesta en los márgenes gingivales.

En el estudio de Gökçe \& Kaya se compararon cuatro grupos de pacientes con distintos tipos de retención fija, en cuanto a grosor y técnicas de adhesión, y un grupo con retención removible tipo Essix. No se encontraron diferencias estadísticamente significativas entre los grupos en cuanto a IP, Recesión Gingival (RG) y Profundidad al Sondaje (PS). Los parámetros de IG y Sangrado AI Sondaje (SAS) con los retenedores fijos se mantuvieron sin variación mientras que mejoraron con el grupo de retenedor Essix, lo que podría significar que éstos permiten realizar higiene oral de manera más eficaz. Se concluye en este estudio que ninguno de los tipos de retención incluidos genera un detrimento de la salud periodontal, no obstante, el tiempo de seguimiento fueron sólo seis meses. Al-Moghrabi et al. (2018) obtuvo conclusiones similares, en el estudio realizaron un seguimiento de 4 años a dos grupos de pacientes usuarios de retenedores mandibulares fijos de canino a canino y tipo Essix. Ambos grupos obtuvieron altos niveles de inflamación gingival y acúmulo de cálculo dentario, pero sin diferencias estadísticamente significativas entre ellos. Este hallazgo confirma que a medida que transcurre el tiempo el paciente va disminuyendo su motivación al percibir que la parte activa del tratamiento ha finalizado, de manera que es fundamental reforzar la técnica de higiene, incentivar al paciente en cada una de sus citas y realizar tratamiento periodontal si corresponde.

Por otra parte, Çifter et al. obtuvieron como resultados en su estudio que con el uso de retenedor tipo Essix, los IP, IG, SAS, IC disminuyeron a través de los 12 meses de seguimiento, lo que significaría la mejoría en la higiene oral debido a la adaptación al paciente al uso de este tipo de retenedor. Sin embargo, hubo pérdida de inserción clínica y aumento de la profundidad al sondaje, no estadísticamente significativa, que fue asociada a microtrauma mecánico producido por el espesor del retenedor, es decir, no de origen inflamatorio. Resultados similares obtuvo Moslemzadeh et al. (2018) cuyo estudio no arrojó diferencias respecto al IG entre pacientes utilizando retenedores removibles tipo Hawley, Essix de $1 \mathrm{~mm}$ y $1.5 \mathrm{~mm}$ de grosor a los seis meses de seguimiento.

Manzon et al. (2018) realizaron un estudio prospectivo en donde se evaluó por seis meses a 70 pacientes divididos en dos tipos de retenedores indicados: Essix $(0.8 \mathrm{~mm})$ y Hawley. El primer grupo obtuvo mayores niveles de IG, IP, y SAS estadísticamente significativos versus el segundo grupo. Las mayores diferencias ocurrieron a los 3 meses de uso para luego disminuir paulatinamente (IP y IC) o desaparecer (IG y SAS). Los 3 meses coinciden con el cambio de régimen de uso de los retenedores de 24 horas diarias a sólo durante la noche. El retenedor Essix al cubrir de manera completa la corona dentaria impide el efecto protector de la saliva, especialmente durante las noches, lo que puede estar relacionado con mayo- 
res niveles de IC y desmineralización del esmalte. Así mismo, también se encontraron acúmulos de cálculo en la superficie interna de este retenedor ya que posee mayores microporosidades y rugosidades en comparación al retenedor Hawley que es más uniforme. Este hecho puede dificultar su limpieza profunda y, por lo tanto, favorecer la colonización bacteriana. Por esta razón siempre deben ser consideradas las características del material que se utilice para su fabricación. Sin embargo, mediante una encuesta los pacientes refirieron mayor comodidad y conformidad al uso de las contenciones tipo Essix.

También ha sido objeto de interés si el tipo de retenedor fijo influye sobre la progresión a enfermedad periodontal. Ferreira et al. (2019) compararon dos tipos de retenedores mandibulares: convencional (fijo sólo a caninos) y Ortho-Flextech (fijo a caninos e incisivos). Ambos fueron utilizados por los pacientes durante seis meses, con un intervalo de 15 días entre los dos tipos de retención. Se obtuvieron mayores niveles de IP, IG e IC para el segundo grupo ya que al estar adherido a todos los dientes anteriores existen más zonas de retención de alimentos y áreas difíciles de higienizar, lo cual también fue referido por los pacientes en una encuesta de satisfacción realizada. Los pacientes correspondían estudiantes de Odontología, en los que podríamos asumir un mejor conocimiento respecto a las técnicas de higiene y sus consecuencias sobre la salud periodontal, lo que puede influir en los resultados.

Knaup et al. (2019) realizó un estudio retrospectivo e investigó la relación entre la formación de biofilm y el material de fabricación de retenedores fijos. Primero, comparó 31 pacientes con retenedores trenzados de acero y 30 pacientes con retenedores de nitinol diseñados digitalmente con tecnología CAD/ CAM. Si bien, todos los pacientes tuvieron algún grado de detrimento de su salud periodontal, hubo diferencias estadísticamente significativas en IP, IG, SAS, PS en favor a los retenedores de nitinol. Solamente en cuanto a recesiones gingivales la diferencia no fue significativa. Luego, en el mismo estudio, se realizó incubación in vitro e intraoral de los diferentes alambres, en donde a las 24 horas se obtuvo menor formación de biofilm en el alambre de nitinol electropulido. Concluyeron que los retenedores de nitinol pueden tener efectos positivos sobre la salud oral, ya que, al ser fabricados digitalmente tienen mayor precisión en su posicionamiento en boca permitiendo disminuir las áreas de retención de placa bacteriana. $\mathrm{Si}$, además, la superficie es refinada con el proceso de electropulido estas propiedades mejoran al dificultar la adhesión bacteriana. Sin embargo, este estudio consideró pacientes en promedio con 7 meses de uso de estos retenedores, lo que podría ser considerado un periodo corto para evaluar daño periodontal.

Un estudio transversal de Corbett et al. (2015) comparó los efectos del uso de retenedores fijos rectos y ondulados, sin encontrarse diferencias significativas entre ambos grupos en cuando a IG, SAS, PAS, RG y volumen de Fluido Gingival Crevicular (FGC). Se asume que ambas técnicas son compatibles con la salud periodontal, sin embargo, los pacientes indicaron mayor comodidad para realizar la limpieza interproximal con los retenedores ondulados ya que el bucle inferior se ubica levemente sobre la papila interdental permitiendo utilizar la seda o hilo dental de forma adecuada.

El análisis del FGC ha demostrado utilidad como herramienta diagnóstica de inflamación periodontal. Este fluido en estado de salud periodontal se encuentra en bajos niveles (Andrew et al.). Eroglu et al. (2019) evaluaron índices periodontales y presencia bacteriana en el FGC asociado a los retenedores fijos, Hawley y Essix. Al seguimiento de 13 semanas no se encontraron diferencias significativas en cuanto a niveles de IP, IG, SAS, PS. Los mismos resultados se obtuvieron respecto a niveles de $S$. Mutans y $L$ Casey, bacterias implicadas en el proceso de desmineralización del esmalte y progresión de la lesión de caries. Todos los pacientes presentaron mejoría de la higiene oral con el tiempo debido a la remoción de la aparatología fija del tratamiento ortodóncico activo.

Por otro lado, Rody Jr. et al. (2016) analizaron el FGC y la presencia de biomarcadores en tres grupos de pacientes con retenedores mandibulares fijos a caninos (grupo I), fijos a caninos e incisivos (grupo II) y removibles no especificado (grupo III), con un mínimo de seis meses de uso. En este estudio no se encontraron diferencias en PS, SAS y volumen de FGC. Sin embargo, el grupo II presentó niveles significativamente mayores de IP, IG, MMP-3, MMP-9 y M-CSF (molécula que influye en el proceso de diferenciación osteoclástica), además niveles disminuidos de OPG, que es un inhibidor de la resorción ósea. Los niveles de RANKL, factor osteoclastogénico, estaban aumentados los grupos I y II con una diferencia estadísticamente significativa. Mayores niveles de ratio RANKL/OPG en grupos I y II pueden estar relacionados una mayor predisposición al desarrollo de enfermedad periodontal, especialmente en pacientes sus- 
ceptibles ya que corresponden a moléculas relacionadas con la actividad osteoclástica (White, 1997). Sin embargo, los parámetros clínicos relacionados a pérdida ósea no se vieron alterados en esta etapa de seguimiento. Creemos que al ser estudio transversal y no contar con grupo control es difícil extrapolar los resultados e inferir que se diferenciarían de un grupo no tratado.

Westerlund et al. realizaron un estudio transversal con tres grupos de pacientes, el primer grupo correspondía a pacientes tratados ortodóncicamente con retención fija por 10 años, el segundo a pacientes tratados sin retención y un tercer grupo control no tratado. Mediante el análisis de Cone Beam Computed Tomography (CBCT) se midió la distancia desde la unión amelocementaria a la cresta marginal del hueso alveolar en todas las superficies de los dientes del grupo V. Se observó que existía perdida de nivel óseo marginal en la cara vestibular en los grupos 1 y 2 en comparación al grupo 3, sin embargo, esta pérdida ósea fue relacionada con factores pre - tratamiento del paciente como son la mordida abierta esqueletal, rotación posterior de la mandíbula, protrusión incisal y extracciones dentarias. El uso de un mecanismo de retención puede causar mayor acúmulo de cálculo dentario, pero, por sí sola no parece ser causante de pérdida de nivel óseo después de 10 años de uso ya que también se presentó en pacientes sin retención.

La pérdida de los tejidos de soporte comienza con la formación de placa dentaria y acumulación de cálculo dentario, sin embargo, como se ha observado no ocurre en todos los pacientes que lo presentan (Kinane). Esto se debe a que el cálculo dentario aumenta la energía superficial para favorecer la colonización bacteriana y no tiene estricta relación a la pérdida ósea (Westerlund et al.). La progresión de la enfermedad periodontal es mutifactorial e involucra tanto factores individuales (respuesta inmune e inflamatoria, enfermedades sistémicas) como factores ambientales (estado socioeconómico, raza, hábitos, técnica de higiene) de cada paciente.

De todas formas, no debe ser despreciado el desarrollo de gingivitis que corresponde a la inflamación de los tejidos blandos alrededor de los dientes (gingiva) y su respuesta inmune a la placa bacteriana (Kinane). En general, una vez retirada la aparatología fija los pacientes presentaban una mejoría en la higiene oral, pero con el tiempo, los índices periodontales volvían a empeorar (Storey et al.) ya que muchas veces, el paciente se desmotiva al creer que la parte activa del tratamiento ortodóncico ha terminado. Por esta razón, debemos recordar la importancia de la motivación constante del paciente con el refuerzo correspondiente de su técnica de higiene oral, por lo menos, cada seis meses. También es nuestro deber educarlo respecto a las consecuencias de no mantener una correcta higiene oral.

Creemos importante que la elección final del tipo de retenedor que sea indicado debe ser en base a múltiples factores que incluyan: la edad, madurez, capacidad de mantener una adecuada higiene oral, biotipo gingival, características oclusales 0 esqueletales previas al tratamiento. Un retenedor fijo se ha recomendado para casos con pérdida ósea aumentada, por la pérdida de estabilidad primaria, o en casos de apiñamiento severo (Ferreira et al.).

En general, los estudios citados reportaron cambios menores en los índices periodontales, sobre todo en cuanto a la pérdida ósea (RG o PS). Creemos que el uso de retenedores presenta mayores beneficios en comparación a los riesgos, siempre y cuando se consideren las distintas variables personales de cada paciente de manera que se asegure la no afectación de los tejidos periodontales.

Cabe recalcar que son pocos los estudios que constan de un tiempo de seguimiento adecuado, que pueda permitir extrapolar los resultados, por lo que es difícil concluir que estos cambios sean temporales o permanentes. Creemos importante en un futuro la planificación de nuevo estudios prospectivos que consideren esta variable. Asimismo, sólo algunos contaban con grupo control lo que podría ayudarnos a comparar los resultados con pacientes no tratados y poder dar validez a los hallazgos en relación con los distintos tipos de retenedores.

\section{CONCLUSIÓN}

La recesión gingival y pérdida ósea es un proceso multifactorial que tiende a aumentar con la edad, el acumulo de cálculo dentario, una técnica incorrecta de higiene y no por la presencia de un retenedor de ortodoncia per se. Así, la motivación transmitida por el ortodoncista y la educación del paciente es un factor esencial para el éxito del tratamiento en el largo plazo. El uso de retenedor de ortodoncia sigue mostrando más beneficios que deterioro sobre la salud 
periodontal; ayudando en la mantención de las posiciones oclusales obtenidas por el tratamiento, evitando la recidiva o nuevas maloclusiones, es por esto, que su elección debe basarse sobre las características individuales de cada paciente.

CORTÍNEZ, L. C.; FIGUEROA, S. M.; CHAMORRO, P. S.; ZAFE, B. F. \& GALLARDO, S. V. Orthodontic retainers and their effect on periodontal health. Literature review. Int. J. Odontostomat., 15(2):460-465, 2021.

ABSTRACT: Due to the high prescription after orthodontic treatment of fixed and removable retainers with an indication of indefinite use, the aim of this study was to find the impact of orthodontic retainers on the periodontal health of the teeth. we proceed to search and analyze scientific articles from databases PubMed, ScienceDirect, SciELO, LILACS and Google Scholar, selected articles were published between 2015 and 2020 based on inclusion and exclusion criteria. Our review suggests that the indication of a fixed or removable retainer presents greater benefits than risks and does not mean, when considering the individual characteristics of each patient, the detriment of periodontal health. However, new prospective studies with a longer followup time and the presence of a control group are necessary to be able to extrapolate results and elucidate the possible damages associated with orthodontic retainers.

KEY WORDS: orthodontic retainers, bone resorption, periodontics.

\section{REFERENCIAS BIBLIOGRÁFICAS}

Al-Moghrabi, D.; Johal, A.; O'Rourke, N.; Donos, N.; Pandis, N.; Gonzales-Marin, C. \& Fleming, P. S. Effects of fixed vs removable orthodontic retainers on stability and periodontal health: 4-year follow-up of a randomized controlled trial. Am. J. Orthod. Dentofacial Orthop.,154(2):167-174.e1, 2018.

Al-Moghrabi, D.; Pandis, N. \& Fleming, P. S. The effects of fixed and removable orthodontic retainers: a systematic review. Prog. Orthod., 17(1):24, 2016.

Çifter, M.; Gümrü Çelikel, A. D. \& Çekici, A. Effects of vacuumformed retainers on periodontal status and their retention efficiency. Am. J. Orthod. Dentofacial Orthop., 152(6):830-5, 2017.

Corbett, A. I.; Leggitt, V. L.; Angelov, N.; Olson, G. \& Caruso, J. M. Periodontal health of anterior teeth with two types of fixed retainers. Angle Orthod., 85(4):699-705, 2015.

Di Venere, D.; Pettini, F.; Nardi, G. M.; Laforgia, A.; Stefanachi, G.; Notaro, V.; Rapone, B.; Grassi, F. R. \& Corsalini, M. Correlation between parodontal indexes and orthodontic retainers: prospective study in a group of 16 patients. Oral Implantol. (Rome), 10(1):78-86, 2017.

Eroglu, A. K.; Baka, Z. M. \& Arslan, U. Comparative evaluation of salivary microbial levels and periodontal status of patients wearing fixed and removable orthodontic retainers. Am. J. Orthod. Dentofacial Orthop., 156(2):186-92, 2019.
Ferreira, L. A.; Sapata, D. M.; Provenzano, M. G. A.; Hayacibara, R. M. \& Ramos, A. L. Periodontal parameters of two types of $3 \times 3$ orthodontic retainer: a longitudinal study. Dental Press J. Orthod., 24(3):64-70, 2019.

Gökçe, B. \& Kaya, B. Periodontal effects and survival rates of different mandibular retainers: comparison of bonding technique and wire thickness. Eur. J. Orthod., 41(6):591-600, 2019.

Highfield, J. Diagnosis and classification of periodontal disease. Aust. Dent. J., 54 Suppl. 1:S11-S26, 2009.

Johnston, C. D. \& Littlewood, S. J. Retention in orthodontics. Br. Dent. J., 218(3):119-22, 2015.

Juloski, J.; Glisic, B. \& Vandevska-Radunovic, V. Long-term influence of fixed lingual retainers on the development of gingival recession: A retrospective, longitudinal cohort study. Angle Orthod., 87(5):658-64, 2017.

Kinane, D. F. Causation and pathogenesis of periodontal disease. Periodontol. 2000, 25:8-20, 2001.

Knaup, I.; Wagner, Y.; Wego, J.; Fritz, U.; Jäger, A. \& Wolf, M. Potential impact of lingual retainers on oral health: comparison between conventional twistflex retainers and CAD/CAM fabricated nitinol retainers : A clinical in vitro and in vivo investigation. J. Orofac. Orthop., 80(2):88-96, 2019.

Manzon, L.; Fratto, G.; Rossi, E. \& Buccheri, A. Periodontal health and compliance: A comparison between Essix and Hawley retainers. Am. J. Orthod. Dentofacial Orthop., 153(6):852-60, 2018.

Moslemzadeh, S. H.; Sohrabi, A.; Rafighi, A. \& Farshidnia, S. Comparison of stability of the results of orthodontic treatment and gingival health between hawley and vacuum-formed retainers. J. Contemp. Dent. Pract. 2018;19(4):443-9, 2018.

Rody Jr., W. J.; Elmaraghy, S.; McNeight, A. M.; Chamberlain, C. A.; Antal, D.; Dolce, C.; Wheeler, T. T.; McGorray, S. P. \& Shaddox, L. M. Effects of different orthodontic retention protocols on the periodontal health of mandibular incisors. Orthod. Craniofac. Res., 19(4):198-208, 2016.

Storey, M.; Forde, K.; Littlewood, S. J.; Scott, P.; Luther, F. \& Kang, $J$. Bonded versus vacuum-formed retainers: a randomized controlled trial. Part 2: periodontal health outcomes after 12 months. Eur. J. Orthod., 40(4):399-408, 2018.

Westerlund A, Oikonomou C, Ransjö M, Ekestubbe A, Bresin A, Lund $\mathrm{H}$. Cone-beam computed tomographic evaluation of the longterm effects of orthodontic retainers on marginal bone levels. Am. J. Orthod. Dentofacial Orthop., 151(1):74-81, 2017.

White, D. J. Dental calculus: recent insights into occurrence, formation, prevention, removal and oral health effects of supragingival and subgingival deposits. Eur. J. Oral Sci., 105(5 Pt. 2):508-22, 1997.

Dirección para correspondencia:

Cristine Cortínez Lira

Llano Subercaseaux 3589

San Miguel

Santiago

CHILE

E-mail: ccortinezlira@gmail.com 